\title{
Hamiltonian formalism for the Oppenheimer-Snyder model
}

\author{
R. Casadio \\ Dipartimento di Fisica, Università di Bologna, and \\ I.N.F.N., Sezione di Bologna, \\ via Irnerio 46, 40126 Bologna, Italy
}

\begin{abstract}
An effective action in Hamiltonian form is derived for a self-gravitating sphere of isotropic homogeneous dust. Starting from the Einstein-Hilbert action for barotropic perfect fluids and making use of the symmetry and equation of state of the matter distribution we obtain a family of reduced actions for two canonical variables, namely the radius of the sphere and its ADM energy, the latter being conserved along trajectories of the former. These actions differ by the value of the (conserved) geodesic energy of the radius of the sphere which defines (disconnected) classes of solutions in correspondence to the inner geometry and proper volume of the sphere. By replacing the (fixed) geodesic energy with its expression in terms of the Schwarzschild time at the surface of the sphere and treating the latter as a further canonical variable we finally obtain an extended action which covers the full space of solutions. Generalization to the (inhomogeneous) Tolman model is shown to be straightforward. Quantization is also discussed.
\end{abstract}

04.60.Ds

\section{INTRODUCTION}

In Ref. [1] the Hamiltonian formalism was developed for general classical barotropic fluids with step- and $\delta$-function discontinuities coupled to Einstein's gravity. In particular, for the case with one step-function discontinuity which separates a region filled with matter from an external asymptotically flat vacuum, the canonical Hamiltonian was shown to consist of one volume contribution plus the Arnowitt-Deser-Misner (ADM) energy which appears as a surface contribution in the asymptotically flat region [2]. No particular symmetry was assumed for the matter distribution.

Subsequently, in Ref. [3] this formalism was applied to the case of a thin shell (with a $\delta$-function discontinuity) by assuming the spherical symmetry from the beginning. Then, adapting to this context a treatment invented for the spherically symmetric vacuum by Kuchar 胿, the action was further reduced to a form which contains only the physical degrees of freedom of the system, that is the shell radius and its ADM energy.

A somewhat simpler example of discontinuous fluid is represented by a sphere of homogeneous dust immersed in the vacuum. This system is known as the Oppenheimer-Snyder (OS) model of gravitational collapse and its Lagrangian equations of motion were solved long ago [5]. However, to our knowledge, a clean and complete derivation from first principles of an action in Hamiltonian form for the OS model which covers the whole space of solutions is missing (see e.g. Refs. 6 [ \& and section $\mathrm{V}$ ) and this is precisely the aim of the present notes. The relevance of such issue is evident if one considers that the construction of a canonical form of the action is an essential step towards the canonical quantization of a dynamical system (this is actually the aim of the above mentioned papers [6 \&]).

The logical scheme we will pursue is essentially the same as in [3]. We start from the general formalism given in [1] and, after having identified all the properties of the matter source, we use such information to partially fix the form of the metric both inside and outside the sphere of dust. We do this in details for the case of homogeneous dust and show that no new degree of freedom arises for the case of inhomogeneous dust with whatever fixed shape for the density profile (Tolman model [9]). The final reduction is gained by applying the transformations defined in [4] to that part of the action corresponding to the outer vacuum and by solving some of the constraints. In so doing we will see the important role played by the known matching conditions at the surface of the sphere from the point of view of Ref. [1] (see Ref. [10] for the standard treatment).

The provisional outcome of our approach is an action in Hamiltonian form which describes the constrained dynamics of two canonical variables representing respectively the radius of the sphere in Schwarzschild coordinates and the ADM energy. The latter is not assumed to be constant nor is dust proper energy. However, the ADM energy is proved to be conserved along the trajectories of the radius determined by the super-Hamiltonian constraint. As a further consequence of the equations of motion, dust proper energy is conserved as well.

So far the super-Hamiltonian constraint coincides with the classical counterpart of the Wheeler-DeWitt equation [11,12] recently used in Refs. [8,21] (see also Ref. [6,7] for a different identification of variables). One also notes that such constraint depends on the (discrete) parameter of the 3-curvature inside the sphere and the (continuous) comoving radial coordinate of the surface of the sphere. These two parameters are invariants (or perennials, see e.g. [13] and Refs. therein) related to the conserved geodesic energy of the radius of the sphere. Therefore one concludes 
that the price to pay for the attained simplification is that the space of OS solutions to Einstein's equations must be divided into classes of solutions with fixed geodesic energy. Then each class can be treated as a separate dynamical system and is assigned an action in canonical form.

Finally we introduce an extended action which describes all the OS solutions. This is done by expressing the geodesic energy as a function of the Schwarzschild time at the surface of the sphere and treating the latter as a further canonical variable together with its conjugate momentum. The final form of the action then contains six canonical variables and one constraint, thus showing that the system possesses two physical degrees of freedom. Of course the transformation to Schwarzschild time is singular at the horizon, but we note that such singularity can be removed from the action by turning to Kruskal (or other regular) coordinates.

In section II we briefly review the relevant results obtained in [1]. In section III we partially fix the metric and $\mathrm{ADM}$ foliation of space-time by inserting all the information defining the matter source. In section IV we derive and interpret the effective actions. In section $\mathrm{V}$ we comment on the quantization of the model and compare to other approaches in the literature.

We use units in which $8 \pi G_{N}=c=1$, Greek indices run from 0 to 3 and Latin indices from 1 to 3 .

\section{HAMILTONIAN FOR A STEP-DISCONTINUOUS FLUID}

To start with, we assume that our space-time manifold $(\Omega, \boldsymbol{g})$ admits a smooth $\left(C^{1}\right)$ foliation into space-like hypersurfaces $S_{t}$ where the parameter $t$ plays the role of time and ranges from $t_{i}$ to $t_{f}$. Then, since we want to describe the dynamics of matter which fills only a compact region of space, it is natural to divide $\Omega$ into the spacetime volume $\Omega_{(-)}$swept by the matter trajectories and its complementary $\Omega_{(+)}=\Omega \backslash \Omega_{(-)}$which are separated by a time-like boundary $\Gamma$. Further $\Omega_{(+)}$has another time-like boundary $\Gamma_{+}$which also bounds $\Omega$ as a whole. Correspondingly $S_{t}$, for each value of $t$, is divided into an internal region $S_{(-)}=\Omega_{(-)} \cap S_{t}$ where matter has support and an external region $S_{(+)}=\Omega_{(+)} \cap S_{t}$ which is empty $\left(S_{t}=S_{(-)} \cup S_{(+)}\right)$. The interior $S_{(-)}$has only one boundary $\partial S_{(-)}:=\Sigma=\Gamma \cap S_{t}$, while the exterior is bounded by both $\Sigma$ and $\Sigma_{+}=\Gamma_{+} \cap S_{t}$, where the latter is a 2 -surface which will eventually be pushed to spatial infinity.

To be more specific, let us introduce a coordinate $r \geq 0$ on $S_{t}$ which is adapted to the boundaries [1] so that the origin $r=0$ is placed inside the matter distribution, $\Sigma_{t}$ is at fixed $r=r_{s}$ and $\Sigma_{+}$at $r=r_{+}>r_{s}$.

In Ref. [i] matter is in the form of a perfect fluid, therefore its energy-momentum tensor can be written (in a set of coordinates $x^{\mu}=\left(t, r, x^{2}, x^{3}\right)$ which cover $\left.\Omega\right)$

$$
T^{\mu \nu}=\sqrt{-g}\left[(e+p) u^{\mu} u^{\nu}+p g^{\mu \nu}\right],
$$

where $e=e\left(x^{\mu}\right)$ is the density, $p=p\left(x^{\mu}\right)$ is the pressure and

$$
u^{\mu}=\frac{d x^{\mu}}{d \tau}
$$

is the 4 -velocity of the fluid which satisfies the usual time-like condition $u_{\mu} u^{\mu}=-1$.

For the case $e=p=0$ in $S_{(+)}$for all values of $t$, it was shown in [1] that the action can be written in the canonical form with Hamiltonian

$$
\mathcal{H}=\int_{S_{t}} d r d x^{2} d x^{3} \sqrt{q}\left(N H+N_{i} H^{i}\right)-\frac{1}{2} \int_{\Sigma_{+}} d x^{2} d x^{3} Q^{t t} \gamma_{t t},
$$

where $\boldsymbol{q}$ is the 3-metric on $S_{t}, q$ its determinant, $N$ the lapse function, $N_{i}$ the shift functions, $\gamma$ the 3-metric on $\Gamma$ and $\boldsymbol{Q}$ the extrinsic curvature of $\Gamma[14$. Further

$$
H:=-\frac{1}{\sqrt{-g}}\left(G^{\mu \nu}-T^{\mu \nu}\right) u_{\mu} u_{\nu},
$$

is the super-Hamiltonian,

$$
H^{i}:=-\frac{1}{\sqrt{-g}}\left(G_{\mu}^{i}-T_{\mu}^{i}\right) u^{\mu}
$$

are the super-momenta and $G_{\mu \nu}$ the Einstein tensor. The surface term at $\Sigma_{+}$in Eq. (2.3) is then proved to equal the ADM energy of the system [2].

We are now ready to simplify the above expressions by making use of the assumed symmetry and equation of state of the matter source. 


\section{SYMMETRY AND EQUATION OF STATE OF MATTER}

The particular matter configuration under study is isotropic, therefore we can assume that $\left(S_{t}, \boldsymbol{q}\right)$ is spherically symmetric. This allows us to give $r$ the meaning of a radial coordinate, with $r=0$ as the centre of the sphere $S_{(-)}$ and take eventually $r_{+} \rightarrow+\infty$. To complete the set of spatial coordinates on $S_{t}$ in a way which is adapted to the symmetry we simply supplement two angles $(\theta, \phi)$. In these coordinates we can then write $e=e(r, t)$ and $p=p(r, t)$.

The shift functions $N_{\theta}$ and $N_{\phi}$ can be set to zero and the 3-metric $\boldsymbol{q}$ on $S_{t}$ can be written without loss of generality

$$
d \sigma^{2}=\Lambda^{2} d r^{2}+R^{2}\left(d \theta^{2}+\sin ^{2} \theta d \phi^{2}\right)
$$

where $\Lambda=\Lambda(r, t)$ and $R=R(r, t)$ are unspecified functions which do not depend on the angular coordinates and which we require to be smooth $\left(C^{1}\right)$ across $\Sigma$ and to have the correct fall-off behaviours for $r=r_{+} \rightarrow+\infty$ [ 4 . In particular we want $\left(S_{t}, \boldsymbol{q}\right)$ to be asymptotically flat (the cosmological constant is set to zero) when its border $\Sigma_{+}$is pushed to spatial infinity, thus

$$
\Lambda(r, t)=1+\frac{m}{r}+\mathcal{O}\left(r^{-2}\right)
$$

where $m$ is the ADM energy.

So far only the spherical symmetry has been used, however our matter source has more nice features. The next step is to recall that the pressure vanishes for dust, $p(e)=0$, therefore Eq. (2.1) simplifies to

$$
T^{\mu \nu}=\sqrt{-g} e u^{\mu} u^{\nu} .
$$

We observe in passing that, as a vector field, $\boldsymbol{u}(r, t)$ is singular at crossing points where the paths of two dust particles meet.

We have still one last piece of information to put in, namely homogeneity. This property can be formulated in the following way: $i$ ) along the streamlines of the particles of dust (with tangent vector $\boldsymbol{u}$ ) the density $e$ is a function of proper time only [17, and $i$ ) the density does not change if we shift the point of inspection perpendicularly to a streamline, that is along any space-like vector $\boldsymbol{m}$ such that

$$
u_{\mu} m^{\mu}=0
$$

More formally the above conditions are written as ( $e$ is a scalar density)

$$
\mathcal{L}_{\boldsymbol{u}} e=u^{\mu} \partial_{\mu} e=f(\tau) \text { and } \mathcal{L}_{\boldsymbol{m}} e=m^{\mu} \partial_{\mu} e=0
$$

where $\mathcal{L}$ is the Lie derivative and $f$ a function of proper time to be determined separately (see section IV]).

Eq. (3.5) could be considered as a set of two partial differential equations which specify $e$ as a functional of the metric $\boldsymbol{g}$ which enters through the condition (3.4) and normalizations. It is instead more useful to assume the existence of a preferred reference frame in which

$$
\mathcal{L}_{\boldsymbol{u}}=\partial_{t}=N \partial_{\tau} \quad \text { and } \quad \mathcal{L}_{\boldsymbol{m}}= \pm \partial_{r}
$$

where the lapse function $N(r, t)=N(t)$ for $0 \leq r<r_{s}$ and we have restricted $\boldsymbol{m}$ according to the spherical symmetry ( $\boldsymbol{m}$ in general is a three dimensional unit vector). Of course in these coordinates the density,

$$
e=e(t)
$$

is a function of time only, as follows from the first equation in (3.5) and the definitions (3.6).

Since the coordinates $r$ and $t$ have to cover smoothly the whole space-time manifold $\Omega$, Eq. (3.6) implicitly requires that the vector fields $\boldsymbol{u}$ and $\boldsymbol{m}$ are regular everywhere in $\Omega$. This rules out a priori all configurations of matter trajectories with crossings between streamlines and $(r, t, \theta, \phi)$ is a comoving frame in which $r$ labels the lines of flow of the particles in the fluid and $t$ parameterizes the position along each streamline. Further, since $\partial_{t}$ is chosen perpendicular to $S_{t}$, then $N_{r}(r, t)=0$ for $0 \leq r<r_{s}$, and the corresponding foliation of space-time inside the matter source is also called time-orthogonal [18].

The choice of Eq. (3.6) and homogeneity also determine the 3-metric $\boldsymbol{q}_{(-)}$in $S_{(-)}$to be given by the unique maximally symmetric Robertson-Walker line elements 15

$$
d \sigma_{-}^{2}=K^{2}\left[\frac{d r^{2}}{1-\epsilon r^{2}}+r^{2}\left(d \theta^{2}+\sin ^{2} \theta d \phi^{2}\right)\right]
$$


where $\epsilon=0, \pm 1$ for respectively flat, spherical and hyperbolic $S_{(-)}, K=K(t)$ is a function of time only and $0 \leq r<r_{s}$ with $r_{s}<1$ for $\epsilon=1$ (we will see in section IV the dynamical meaning of $\epsilon$ and $r_{s}$ ).

In $S_{(+)}$the density $e=0$ and no comoving frame can be defined. Therefore the 3-dimensional metric $\boldsymbol{q}_{(+)}$cannot be a priori reduced to a form simpler than the one given in Eq. (3.1). However, at the surface $\Sigma$ one still has to enforce suitable matching conditions such that $\left(S_{t}, \boldsymbol{q}\right)$ is a $C^{1}$ metric manifold as prescribed.

For the pull-back of the 4-dimensional metric $\boldsymbol{g}$ on $S_{t}$ to be $C^{1}$ one needs $\boldsymbol{q}_{(-)}$, Eq. (3.8), and $\boldsymbol{q}_{(+)}$, Eq. (3.1), to satisfy

$$
\begin{aligned}
& \left.\Lambda\right|_{+}=\left.\frac{K}{\sqrt{1-\epsilon r^{2}}}\right|_{-} \\
& \left.\dot{\Lambda}\right|_{+}=\left.\frac{\dot{K}}{\sqrt{1-\epsilon r^{2}}}\right|_{-} \\
& \left.\Lambda^{\prime}\right|_{+}=\left.\frac{K \epsilon r}{1-\epsilon r^{2}}\right|_{-}
\end{aligned}
$$

and

$$
\begin{aligned}
& \left.R\right|_{+}=\left.K r\right|_{-} \\
& \left.\dot{R}\right|_{+}=\left.\dot{K} r\right|_{-} \\
& \left.R^{\prime}\right|_{+}=K,
\end{aligned}
$$

where we have introduced the notations $\left.A\right|_{ \pm}:=\lim _{\delta \rightarrow 0} A\left(r_{s} \pm \delta, t\right), \dot{A}:=\partial_{t} A$ and $A^{\prime}:=\partial_{r} A$ for any function $A$.

In the above we have assumed $\boldsymbol{g}$ is written in the usual ADM form with lapse function $N$ and shift functions $N_{i}$ [14]. Of the latter only the radial one, denoted by $N_{r}$, survives after the imposition of the spherical symmetry. Moreover, we have just seen that in $S_{(-)}$one has $N=N(t)$ because of homogeneity and $N_{r}=0$ because $S_{(-)}$is a time-orthogonal foliation. Thus, on $\Sigma$

$$
\begin{aligned}
& \left.N\right|_{+}=N(t) \\
& \left.\dot{N}\right|_{+}=\left.\dot{N}\right|_{-} \\
& \left.N^{\prime}\right|_{+}=0
\end{aligned}
$$

and

$$
\left.N_{r}\right|_{+}=\left.\dot{N}_{r}\right|_{+}=\left.N^{\prime}\right|_{+}=0 .
$$

The matching conditions (3.9) and (3.10) will turn out to be extremely powerful in simplifying the final form of the action.

Let us finish the section with a quick look at the slightly more general case in which

$$
e=f(t) h(r)
$$

in the time-orthogonal comoving frame (which is again supposed to exist). This represents inhomogeneous dust with a fixed density profile that can only rescale in time and is the matter content of the solutions found by Tolman [9. Their metric can be written as in Eq. (3.8), but with $K=J(r) L(t)$ now being also a function of the position inside $S_{(-)}$,

$$
h=2 \frac{\left(r^{2} J\right)^{\prime}}{J^{\prime} J^{2}} .
$$

It is apparent that Eq. (3.7) is a special case of Eq. (3.13) with $h$ constant $(J \propto r)$. Since $h(r)$ is fixed once and for all, there is indeed no more freedom in this generalization with respect to the homogeneous case, thus we will not consider explicitly the density distribution (3.13) in the following sections. 


\section{HAMILTONIAN DYNAMICS}

In the previous section the 3-metric inside the sphere of dust, $S_{(-)}$, has been uniquely fixed to the form in Eq. (3.8) for $0 \leq r<r_{s}$ by making use of all the properties assumed for the matter source. Only isotropy survives in the outer empty region, $S_{(+)}$, and this has led to the more generic form in Eq. (3.1) for $r>r_{s}$ and to the set of matching conditions in Eqs. (3.9)-(3.12).

Plugging in all the above information into the Hamiltonian in Eq. (2.3) gives the action

$$
I=I_{(-)}+I_{(+)}+I_{\Sigma_{+}} .
$$

The first (volume) contribution comes from $S_{(-)}$and is given by

$$
I_{(-)}=12 \pi V_{s} \int_{t_{i}}^{t_{f}} d t\left(P_{K} \dot{K}-N H\right)
$$

The "parametric volume" of the sphere

$$
V_{s}=\int_{0}^{r_{s}} \frac{r^{2} d r}{\sqrt{1-\epsilon r^{2}}}
$$

has been integrated out (along with a factor of $4 \pi$ which we shall always factor out for convenience) and

$$
H:=-\frac{1}{2}\left(\frac{P_{K}^{2}}{K}+\epsilon K-\frac{2}{3} e K^{3}\right)
$$

is the internal super-Hamiltonian which contains dust proper energy,

$$
H_{M}:=V_{s} \text { e } K^{3},
$$

and the momentum conjugated to $K$ is defined by

$$
P_{K}:=-\frac{K \dot{K}}{N}
$$

The second (volume) contribution for $S_{(+)}$can be read straightforwardly from Ref. 㺻,

$$
I_{(+)}=4 \pi \int_{t_{i}}^{t_{f}} d t \int_{r_{s}}^{+\infty} d r\left(P_{\Lambda} \dot{\Lambda}+P_{R} \dot{R}-N H_{(+)}-N^{r} H_{r}\right),
$$

with

$$
\begin{aligned}
& H_{(+)}:=-\frac{P_{R} P_{\Lambda}}{R}+\frac{\Lambda P_{\Lambda}^{2}}{2 R^{2}}+\frac{R R^{\prime \prime}}{\Lambda}-\frac{R R^{\prime} \Lambda^{\prime}}{\Lambda^{2}}+\frac{R^{\prime 2}}{2 \Lambda}-\frac{\Lambda}{2} \\
& H_{r}:=P_{R} R^{\prime}-\Lambda P_{\Lambda}^{\prime},
\end{aligned}
$$

and

$$
\begin{aligned}
& P_{R}:=-\frac{R}{N}\left(\dot{R}-N^{r} R^{\prime}\right) \\
& P_{\Lambda}:=-\frac{\Lambda}{N}\left(\dot{R}-N^{r} R^{\prime}\right)-\frac{R}{N}\left(\dot{\Lambda}-\left(N^{r} R\right)^{\prime}\right) .
\end{aligned}
$$

As a very simple check one can see that from Eqs. (3.9) and (3.10) it follows that $\left.H_{r}\right|_{+}=0$ and

$$
\left.H_{(+)}\right|_{+}=\frac{1}{3 V_{s}} \frac{r_{s}^{2}}{\sqrt{1-\epsilon r_{s}^{2}}}\left(\left.H_{(-)}\right|_{-}-3 e K^{3}\right)
$$

so that the super-Hamiltonian encodes the (step-function) discontinuity at $r=r_{s}$.

Finally, the (surface) contribution 


$$
I_{\Sigma_{+}}=-4 \pi \int_{t_{i}}^{t_{f}} d t N_{+} m
$$

contains the ADM energy and the lapse function $N_{+}:=N\left(r_{+}, t\right)$.

The internal action $I_{(-)}$is already in its simplest possible form. The external action $I_{(+)}$instead can be greatly simplified by the following change of canonical variables [4]. We define a new momentum conjugated to $R$ :

$$
\bar{P}_{R}:=P_{R}-\left(\frac{1}{F_{+} F_{-}}\right) \frac{\Lambda P_{\Lambda}}{2 R}-\frac{R}{2}\left(\ln \left|\frac{F_{+}}{F_{-}}\right|\right)^{\prime},
$$

where $F_{ \pm}:=R^{\prime} / \Lambda \pm P_{\Lambda} / R$, and introduce two new canonically conjugated variables,

$$
\begin{aligned}
& M:=\frac{P_{\Lambda}^{2}}{2 R}-\frac{R R^{\prime 2}}{2 \Lambda^{2}}+\frac{R}{2} \\
& P_{M}:=\frac{\Lambda P_{\Lambda}}{R F_{+} F_{-}} .
\end{aligned}
$$

The difference between the corresponding Liouville forms 沟]

$$
\Delta:=\bar{P}_{R} \delta R+P_{M} \delta M-P_{R} \delta R-P_{\Lambda} \delta \Lambda=\delta(\omega)+\left(\delta R^{2} \ln \left|\frac{F_{+}}{F_{-}}\right|\right)^{\prime},
$$

once integrated from $r=r_{s}$ to $r=r_{+} \rightarrow+\infty$ contributes at most a surface term at $r=r_{s}(\delta(\omega)$ can be discarded since it corresponds to boundary terms at $t=t_{i}$ and $t=t_{f}$ which do not change the equations of motion). By applying the matching conditions (3.9), (3.10) it is easy to see that

$$
\int_{r_{s}}^{+\infty} d r \Delta=-R^{2} \delta \ln \left|\frac{F_{+}}{F_{-}}\right|_{+}=\Phi(K, \dot{K}) \delta \dot{K} .
$$

Therefore $\Delta$ does not contribute to $I_{(+)}$and we can map $\left(R, P_{R} ; \Lambda, P_{\Lambda}\right)$ to $\left(R, \bar{P}_{R} ; M, P_{M}\right)$ without getting any new term in the action. We point out that this is a major consequence of the smoothness of $S_{t}$ across $\Sigma[10]$.

In $S_{(+)}$the new momentum conjugated to $R$ and $M^{\prime}$ are linear combinations of the constraints $H_{(+)}$and $H_{r}$ 沺,

$$
\begin{aligned}
\bar{P}_{R} & =\frac{1}{F_{+} F_{-}}\left(\frac{P_{\Lambda}}{R} H_{(+)}+\frac{R^{\prime}}{\Lambda^{2}} H_{r}\right) \\
M^{\prime} & =-\frac{1}{\Lambda}\left(R^{\prime} H_{(+)}+\frac{P_{\Lambda}}{R} H_{r}\right),
\end{aligned}
$$

and vanish along classical solutions. Then one can substitute $I_{(+)}$with

$$
\bar{I}_{(+)}=4 \pi \int d t \int_{r_{s}}^{+\infty} d r\left(P_{M} \dot{M}+\bar{P}_{R} \dot{R}-N^{R} \bar{P}_{R}-N^{M} M^{\prime}\right),
$$

where the new Lagrange multipliers $N^{R}$ and $N^{M}$ can be expressed in terms of $N$ and $N^{r}$ 国.

At this point we are ready to reduce the action. First we solve the constraints

$$
\bar{P}_{R}=0, \quad M^{\prime}=0
$$

and substitute the solutions back into $\bar{I}_{(+)}$. Then we rename $N_{+}:=-\dot{T}$ and get the reduced action

$$
\begin{aligned}
I & =12 \pi V_{s} \int_{t_{i}}^{t_{f}} d t\left(P_{K} \dot{K}-N H\right)+4 \pi \int_{t_{i}}^{t_{f}} d t\left(\dot{M} \int_{r_{s}}^{+\infty} d r P_{M}+\dot{T} m\right) \\
& =12 \pi V_{s} \int_{t_{i}}^{t_{f}} d t\left[P_{K} \dot{K}+p \dot{m}+\frac{N}{2}\left(\frac{P_{K}^{2}}{K}+\epsilon K-\frac{2 m}{r_{s}^{3}}\right)\right],
\end{aligned}
$$

where in the last line we have inferred from $M^{\prime}=0$ that 


$$
m:=\left.M\right|_{+}=\frac{r_{s}^{3}}{2}\left(\frac{P_{K}^{2}}{K}+\epsilon K\right),
$$

and this, together with $H=0$, relates the ADM energy to the matter proper energy $H_{M}$, Eq. (4.5), according to

$$
m=\frac{r_{s}^{3}}{3} \text { e } K^{3}:=\frac{r_{s}^{3}}{3 V_{s}} H_{M}
$$

where $r_{s}^{3} / 3 V_{s}$ is the ratio between the proper volume of the sphere and the volume of a flat sphere of equal radius [16]. We have also defined the momentum $p$ canonically conjugated to $m$ as

$$
p:=\frac{1}{3 V_{s}}\left(\int_{r_{s}}^{+\infty} d r P_{M}-T\right) .
$$

The time has come to clarify the dynamical meaning of the two parameters $\epsilon$ and $r_{s}$ in the OS model. In order to do this, we define two new canonical variables $R_{s}:=r_{s} K, P_{s}:=P_{K} / r_{s}$ and rewrite the action (4.19) as (we omit an irrelevant overall factor and make use of the arbitrariness of the lapse function)

$$
I_{r_{s}}^{\epsilon}\left(R_{s}, m ; P_{s}, p\right)=\int_{t_{i}}^{t_{f}} d t\left[P_{s} \dot{R}_{s}+p \dot{m}-N H_{r_{s}}^{\epsilon}\right]
$$

with

$$
H_{r_{s}}^{\epsilon}=-\frac{1}{2}\left(\frac{P_{s}^{2}}{R_{s}}+\epsilon r_{s}^{2} R_{s}-2 m\right) .
$$

The super-Hamiltonian constraint then becomes $(d \tau:=N d t$ is the proper time at the surface of the sphere)

$$
\left(\frac{d R_{s}}{d \tau}\right)^{2}=-\epsilon r_{s}^{2}+\frac{2 m}{R_{s}}
$$

which is the expected equation of radial geodesic motion of the radius of the sphere $R_{s}$ in the external Schwarzschild metric with mass parameter [16]

$$
m=\int_{0}^{R_{s}} e R^{2} d R
$$

and geodesic energy ( $T_{s}$ is the Schwarzschild time at the surface of the sphere),

$$
E:=\left(1-\frac{2 m}{R_{s}}\right) \frac{d T_{s}}{d \tau},
$$

given by

$$
E^{2}=1-\epsilon r_{s}^{2}
$$

One observes that for $\epsilon= \pm 1$ the function $E\left(\epsilon, r_{s}\right)=E\left( \pm r_{s}^{2}\right)$ is continuous in $r_{s}$, but for $\epsilon=0$ the value of $r_{s}$ is totally arbitrary, since $E\left(0, r_{s}\right)=1, \forall r_{s}>0$.

In the expressions above the mass parameter is a positive quantity and the geodesic energy ranges from -1 to $+\infty$ $\left(-1 \leq E<1 \Leftrightarrow \epsilon=+1 \Leftrightarrow H_{M} / m>1\right.$ for bound orbits and $E \geq 1 \Leftrightarrow \epsilon=0,-1 \Leftrightarrow H_{M} / m \leq 1$ for unbound orbits [16]). Thus the quantities $E$ and $m$ define separate classes [E, $m$ ] of OS space-time solutions to the Einstein equations, each of which (provided is not empty) can be treated as one constrained dynamical system with canonical variables $R_{s}$ and $P_{s}$. This implies that each trajectory belonging to a given class $[E, m]$ is specified by the initial conditions (at $\tau=\tau_{i}$ )

$$
\left\{\begin{array}{l}
R_{s}\left(\tau_{i}\right):=R_{0}=\left(\frac{3 m}{e\left(\tau_{i}\right)}\right)^{1 / 3} \\
\left(\frac{d R_{s}}{d \tau}\right)_{\tau=\tau_{i}}:=V_{0}= \pm \sqrt{\frac{2 m}{R_{0}}-1+E^{2}}
\end{array}\right.
$$


From the second equation in (4.29) it follows that, in each $[E, m], V_{0}$ admits just two opposite values (if any) once $R_{0}$ is given. Further, from the first equation in (4.29) one sees that the choice of $R_{0}$ (or $V_{0}$ ) is equivalent to fixing the dust proper energy density $e\left(\tau_{i}\right)$ which has totally disappeared from the action.

In these notes, following the basic idea of fully employing the symmetry and equation of state of the matter source but no further information, we have ended up in considering the larger disconnected classes of space-time solutions defined solely by the geodesic energy $E$, that is $[E]=\cup_{m>0}[E, m]$. Therefore in our approach the ADM mass and its conjugate momentum have become canonical variables along with $R_{s}$ and $P_{s}$ and each trajectory in a given class $[E]$ is specified by the initial conditions $R_{0}, V_{0}, m_{0}:=m\left(\tau_{i}\right)$ and $v_{0}:=\dot{m}\left(\tau_{i}\right)$.

In each $[E]$ the allowed values of $R_{0}$ and $V_{0}$ are not uniquely related. However, by varying the action in Eq. (4.19) with respect to $p$ one obtains $\dot{m}=0$, thus $v_{0}=0$ and the ADM energy is conserved $\left(m=m_{0}\right)$ as a consequence of the equations of motion. Then, from Eq. (4.21), one deduces the conservation of matter proper energy as a consequence of the conservation of $m$. At this point Eqs. (4.29) relate $R_{0}$ and $V_{0}$ according to the subclass $\left[E, m_{0}\right]$.

We also observe that Eq. (4.21) does not depend on the internal geometry. In fact, the independence of the ADM energy from $\epsilon$ (internal 3-curvature) is another well known property of the OS model [16] related to Birkhoff's theorem [15.

From the definition of $E$ in Eq. (4.27) and Eq. (4.28) one can rewrite the term $\epsilon r_{s}^{2}$ in the super-Hamiltonian (4.24) as a function of the Schwarzschild time $T_{s}$ which becomes a canonical variable with conjugate momentum

$$
P_{T}=-\left(1-\frac{2 m}{R_{s}}\right)^{2} \frac{R_{s} \dot{T}_{s}}{N}
$$

The extended action thus obtained finally reads

$$
I^{e}\left(T_{s}, R_{s}, m ; P_{T}, P_{s}, p\right)=\int_{t_{i}}^{t_{f}} d t\left[P_{T} \dot{T}_{s}+P_{s} \dot{R}_{s}+p \dot{m}-N H^{e}\right]
$$

where the extended super-Hamiltonian is

$$
H^{e}=-\frac{1}{2}\left(\frac{P_{s}^{2}}{R_{s}}-\frac{R_{s} P_{T}^{2}}{\left(R_{s}-2 m\right)^{2}}+R_{s}-2 m\right) .
$$

Since no parameters but only six canonical variables appear in the expression of $H^{e}$, one concludes that $I^{e}$ describes all OS solutions in the phase space $\mathcal{F}=\cup_{E \geq-1}[E]$. There is still one constraint, thus the system admits two physical degrees of freedom. In this respect, the extended action $I^{e}$ and the super-Hamiltonian $H^{e}$ represent the main results of these notes.

Let us finish the section with two remarks. The reduced action $I_{r_{s}}^{\epsilon}$ is regular for all values of $R_{s}$. On the contrary, since $T_{s}$ diverges at the horizon, the extended action is singular for $R_{s}=2 \mathrm{~m}$. However one can eliminate such singularity by changing from the Schwarzschild coordinates $R_{s}$ and $T_{s}$ to the Kruskal (or other regular and possibly more convenient) coordinates of the surface of the sphere (we leave this analysis to future developments). Finally, the generalization to the case of inhomogeneous dust defined by Eq. (3.13) is straightforward and essentially amounts to changes in numerical coefficients appearing in the action.

\section{REMARKS ON QUANTIZATION}

The main reason of interest in the canonical formalism of the OS model relies in the hope that its quantization might supply a better understanding of the physics of gravitational collapse.

Although very simple in its final form, our extended super-Hamiltonian constraint $H^{e}=0$ contains several difficulties along the path of quantization. Not only the kinetic term leads to the problems of operator ordering and Hermiticity of $\hat{H}^{e}$ but, since $H^{e}$ has non-vanishing Poisson brackets with $T_{s}, R_{s}$ and their momenta, the latter canonical variables cannot even be mapped into quantum observables straightforwardly. Let us however assume that this can be solved by eliminating them in favour of related perennials (see 13 and Refs. therein), as is already done for $T_{s}$ and $P_{T}$ when considering $H_{r_{s}}^{\epsilon}$, and focus on some aspects of the OS model treated in the literature.

One of the first attempts to quantization is found in Lund [6], where dust is represented by a homogeneous scalar field $\varphi$, which serves as a potential for the dust 4-velocity $\left(u^{\mu}:=\partial^{\mu} \varphi\right)$, and its conjugate momentum $P_{\varphi}$. The scalar field, because of the normalization $u_{\mu} u^{\mu}=-1$, has a matter super-Hamiltonian $H_{M}=P_{\varphi}$ and can be used to define a time variable $(t:=-\varphi)$ in order to turn the super-Hamiltonian constraint $H_{G}+H_{M}=0$ into a Schrödinger-like equation $\left[\hat{P}_{\varphi}+\hat{H}_{G}\right] \Psi=0$. The latter does not suffer of the operator ordering ambiguity but cannot be solved 
exactly. The wavefunction $\Psi=\Psi(t, x)$ depends on the quantized canonical variable $x$ related to $R_{s}$ by a canonical transformation which also maps $H_{G}$ into the gravitational part $\left(H_{r_{s}}^{\epsilon}-H_{M}\right)$ of the super-Hamiltonian in our reduced action (provided $m$ is fixed).

The outer metric as well as the matching conditions with the inner metric at the surface of the sphere are never considered explicitly in [6] and the relation between the ratio $3 V_{s} / r_{s}^{3}$ and the geodesic energy $E$ is thus missing. Further, neither the ADM energy is discussed nor the ADM surface term is included so that the interpretation of the reduced action as describing (classes $[E, m]$ of) the OS model, rather than a cosmological model, is incomplete.

On a different ground, one observes that the identification $t:=-\varphi$ prevents the second quantization of the matter content of the model and one cannot even count the number of dust particles. This seems unsuitable for an astrophysical model in which one might rather prefer to study quantized matter on a (semi-)classical background prior to quantization of gravity.

More recently in Refs. [7,8] the super-Hamiltonian in Eq. 4.4 is used and the matching conditions at $r=r_{s}$ are correctly implemented, although somewhat ad hoc, with $m$ constant. However the ADM surface term is again neglected and the partition of the phase space into classes $[E, m]$ is not acknowledged. Moreover, Peleg [7] then goes back to the same action of Ref. [6], in order to avoid the operator ordering ambiguity, and solves the Schrödinger equation with dust as time in the semi-classical (WKB) approximation.

In Ref. [8] the scalar field is not normalized and its (second) quantization gives a proper energy $\left\langle\hat{H}_{M}\right\rangle$ proportional to the number of dust quanta in such a way that the latter is not a priori constant. In this approach $K$ is treated semi-classically and plays the role of time (see Ref. 19] and Refs. therein for the general formalism).

When the scalar field is not normalized one must be careful in asserting that it models dust since it is indeed equivalent to a perfect fluid with pressure equal to its Lagrangian density [20], $p \sim \frac{1}{2}\left(\dot{\phi}^{2}-\mu^{2} \phi^{2}\right)$. However we observe that, if the scalar field has mass $m_{\phi}=\hbar \mu$, then the pressure oscillates with frequency $\sim 2 \mu$ and for $m_{\phi} \sim m_{p} \sim 10^{-27} \mathrm{~kg}$ (the proton mass) this means a period $T \sim 10^{-23} \mathrm{~s}$ (in the classical limit $\hbar \rightarrow 0$ with $m_{\phi}$ constant $T \rightarrow 0$ ). It is thus reasonable to approximate the actual value of $p$ with its average over one period (that is zero) provided $K$ does not change appreciably on the time scale $T$ (adiabatic approximation). In Ref. [8] it was verified that in this approximation $\left(\dot{R}_{s} / R_{s} \ll 1\right)\left\langle\hat{H}_{M}\right\rangle$ is constant and the OS classical trajectories for dust are recovered. This in turn guarantees a posteriori the identification of the quantized scalar field (mode) with dust.

In the present approach neither a scalar field nor other models for the dust are used, leaving room for future developments and possible overlappings with the cited papers. On the other side the matching conditions are implemented from the onset and need not to be imposed by hand later. Further, the ADM surface term is also included from the start and correspondingly the ADM energy is not a priori fixed.

A further improvement of our approach with respect to the cited papers is that our reduced actions $I_{r_{s}}^{\epsilon}$ describe larger classes of OS solutions and the extended action $I^{e}$ covers all the OS solutions. This is no real improvement for the classical theory, since there one only needs to describe one trajectory at a time (not even one class), but is important in the quantized theory, as can be seen already from the following semi-classical argument. Since dust proper energy is not a priori conserved, one could have a non-vanishing probability for the transition from a state with $H_{M}$ corresponding to a certain (semi-)classical trajectory in $[E, m]$ to a different $\bar{H}_{M}$, with $m$ held constant. From (4.21) and the first equation in (4.29) one can then interpret this as a quantum transition to a (semi-classical) trajectory with different comoving radius $\bar{r}_{s}$. The latter trajectory belongs to a different class $[\bar{E}, m]$ and is described by the super-Hamiltonian $H_{\bar{r}_{s}}^{\epsilon}$ such that Eq. (4.21) still holds for the same value $m$ of the ADM energy. We note in passing that such change in $r_{s}$ represents a "motion" of the surface of the sphere with respect to the comoving frame and would thus correspond to the presence of effective (quantum) pressure (see e.g. [22]). If, according to Ref. [21], $\left\langle\hat{H}_{M}\right\rangle$ steadily increases in time, then $E$ converges to 1 and the class $[E=1]$ becomes a sort of quantum attractor in the space of semi-classical states of the OS model.

In a full quantum context one can go even further and imagine a state which is a superposition of several $m$ eigenstates (belonging to the same $[E]$ or not). Such a state is allowed by the indetermination principle applied to the canonical variables $m$ and $p$ and would represent a superposition of space-times where asymptotic $\left(r \gg r_{s}\right)$ trajectories of test particles move according to a "superposition" of Newton laws.

\section{CONCLUSIONS}

In these notes we have carried on a derivation of canonical actions for the OS model which takes into account from the onset the matching conditions at the surface of the sphere and the ADM surface term. This allowed us to identify the range of applicability of the actions already found in the literature and extend them to cover the full space of OS solutions. 
In particular we have shown that the full phase space of the model is naturally parted into classes defined by the geodesic energy of the surface of the sphere which uniquely specifies the inner geometry. We have then introduced actions describing classes of solutions which are larger than the classes obtained by fixing the ADM energy and the geodesic energy, as we have shown that was (implicitly) done in previous papers [6] 8$]$.

We think the above results are useful for a better understanding of the quantization of the OS model as a constrained dynamical system.

\section{ACKNOWLEDGMENTS}

I would like to thank P. Hajicek for suggesting the problem and many useful discussions and the Institute for Theoretical Physics of the University of Bern for the kind hospitality during February 1998.

[1] P. Hajicek and J. Kijowski, Phys. Rev. D 57, 914 (1998).

[2] J. Kijowski, Gen. Rel. Grav. 29, 307 (1997); in Proceedings of Journees Relativistes 1983 (Pitagora Editrice, Bologna, 1985), pp. 205-219.

[3] P. Hajicek, Phys. Rev. D 57, 936 (1998).

[4] K. Kuchar, Phys. Rev. D 50, 3961 (1994).

[5] J. R. Oppenheimer and H. Snyder, Phys. Rev. 56, 455 (1939).

[6] F. Lund, Phys. Rev. D 8, 3253 (1973).

[7] Y. Peleg, Phys. Lett. B 356, 462 (1995)

[8] R. Casadio and G. Venturi, Class. Quantum Grav. 13, 2715 (1996).

[9] R. C. Tolman, Proc. Nat. Acad. Sci. 20, 169 (1934).

[10] W. Israel, Nuovo Cimento 44 B, 1 (1966).

[11] B. S. DeWitt, Phys. Rev. 160, 1113 (1967).

[12] J. A. Wheeler, in Batelle rencontres: 1967 lectures in mathematics and physics edited by C. DeWitt and J. A. Wheeler (Benjamin, New York, 1968)

[13] P. Hajicek, Nucl. Phys. B (Proc. Suppl.) 57, 115 (1997).

[14] R. Arnowitt, S. Deser and C. W. Misner, in Gravitation: an introduction to current research edited by L. Witten, (Wiley, New York, 1962).

[15] S. Weinberg, Gravitation and cosmology (Wiley, New York, 1972).

[16] H. Stephani, General Relativity (Cambridge Univ. Press, Cambridge, 1990)

[17] We do not enforce the conservation of matter proper energy a priori.

[18] In principle one can define a comoving frame which is not time-orthogonal, but this would lead to unnecessary complications, such as the need for a non-vanishing radial lapse function.

[19] C. Bertoni, F. Finelli and G. Venturi, Class. Quantum Grav. 13, 2375 (1996).

[20] M. S. Madsen, Class. Quantum Grav. 5, 627 (1988).

[21] R. Casadio, F. Finelli and G. Venturi, Adiabatic invariant treatment of a collapsing sphere of quantized dust (1997), preprint gr-qc/9706074.

[22] J. V. Narlikar, Introduction to cosmology (Cambridge Univ. Press, Cambridge, 1993). 